SIGNALLING

\section{Fuzzy logic}

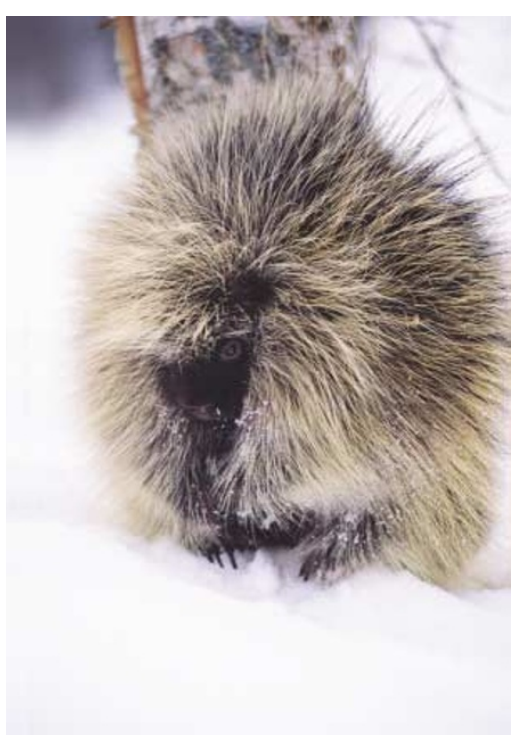

Medulloblastomas are childhood tumours of cerebellar origin that are, in some cases, associated with inappropriate activation of the Hedgehog (Hh) signalling pathway. Philip Beachy and colleagues now report that inhibitors of this pathway induce regression of established tumours in preclinical models of medulloblastoma, revealing a new therapeutic approach for this aggressive cancer.

Hh encompases a family of secreted proteins that are essential for developmental patterning events, as well as for the maintenance of somatic stem cells and specification of organ size. Hh binds to the transmembrane receptor Patched (Ptch), allowing activation of another transmembrane protein named Smoothened (Smo). This leads to the activation of target genes by the Cubitus interruptus/Gli (CI/GLI) family of transcription factors.

Ligand-independent activation of this pathway has been shown to occur in medulloblastoma, caused either by mutations that render Smo insensitive to regulation by Ptch, or by mutational inactivation of Ptch. The absence of Ptch activity can promote tumour initiation, and subsequent loss of p53 function seems to promote tumourcell growth in a mouse model of medulloblastoma.

Beachy and colleagues therefore decided to test the ability of cyclopamine, a plant-derived product that inhibits Hh signalling at the level of Smo, to slow medulloblastoma growth. They found that treatment of meduloblastoma-derived cell lines with cyclopamine inhibited prolifera- tion and induced differentiation in culture. Furthermore, subcutaneous injection of cyclopamine into a mouse model of medulloblastoma created by disruption of one Ptch allele and mutation of Trp53 (which encodes $\mathrm{p} 53$ in mice) — reduced the growth of established tumours.

Cells from freshly resected human medulloblastomas, which were found to have elevated Hh signalling activity, were also susceptible to a derivative of cyclopamine. Treatment with this drug reduced the viability of medulloblastoma cells by almost $50 \%$, and downregulated the Hh-signalling pathway. Cyclopamine, however, had no effect on glioblastoma, ependymoma or fibroblast cells, indicating that it is not a general cytotoxin. Nor was the drug observed to have toxic effects on rodents or other mammals.

So why is Hh-pathway activation a characteristic of medulloblastomas,

\title{
Welcome interference
}

Double-stranded RNA molecules that are specifically designed to block gene expression - termed short interference RNA (siRNAs) - promise to be a useful tool in cancer research. Less than one year after the first demonstration of how siRNAs could silence gene expression in mammalian cell culture, Agami and colleagues, reporting in Cancer Cell, show that oncogenic KRAS can be specifically and stably inactivated through the use of a viral RNA interference vector.

The guanine-nucleotide-binding proteins that are encoded by $R A S$ are integral to cellular signal-transduction pathways. They regulate proliferation, differentiation and cell survival, and are frequently mutated in human cancers, particularly in pancreatic ( $85 \%)$ and colon carcinomas (40\%). Mutant RAS oncogenes often contain point mutations, such as glycine to valine at codon $12\left(K R A S^{\mathrm{v} 12}\right)$, and these lead to constitutively active RAS proteins. Wild-type KRAS seems to be required for viability, and antisense strategies to KRAS - which cannot distinguish between the wild-type and oncogenic forms of the gene - have not been successful.

Brummelkamp et al. used a retroviral vector (pRETROSUPER) carrying the expression cassette of the plasmid pSUPER - developed recently by the same group - that directs the synthesis of siRNAs in mammalian cells. When a human pancreatic cell line (CAPAN1) was infected with the PRETROSUPER vector that contained sequences that spanned the mutation region of $K R A S^{\mathrm{V} 12}$, expression of $K R A S^{\mathrm{V} 12}$ was inhibited. This suppression was specific - use of a TP53 sequence in the vector did not cause a decrease in KRAS. Infecting the EJ bladder cell line - which has mutated HRAS but wild-type KRAS - with the mutant $K R A S$ vector also had no effect on the expression of KRAS.

In addition, the authors showed that the specific suppression of $K R A S^{V 12}$ leads to loss of tumorigenicity. When KRAS $S^{\mathrm{V} 12}$ expression was downregulated in CAPAN-1 cells, the cells could not form tumours in nude mice, whereas mice injected with CAPAN- 1 cells that had been infected with a control vector all developed tumours.
So, how can we make use of viral RNA interference vectors in cancer research? They are a powerful tool for investigating the genetic events that are required for a tumorigenic phenotype and for further elucidating the events that are needed to maintain that phenotype. In addition, siRNA-based gene therapy - shown to be a possibility by Brummelkamp et al. in both cell culture and animal models - should be investigated further. Issues that will need to be addressed include overcoming the poor penetration of tumours, possible neutralization of siRNA by the immune system and the possibility of escape from siRNA-mediated attack.

Ezzie Hutchinson

(4) References and links ORIGINAL RESEARCH PAPER Brummelkamp, T. R. et al. Stable suppression of tumorigenicity by virus-mediated RNA interference. Cancer Cel/ 2, 243-247 (2002)

FURTHER READING Borkhardt, A. Blocking oncogenes in malignant cells by RNA interference - new hope for a highly specific cancer treatment. Cancer Cel/ 2, 167-168 (2002) | Tuschl, T. Expanding small RNA interference. Nature

Biotechnol. 20, 446-448 (2002)

WEB SITE

Reuven Agami's lab: http://wwww.nki.n//nkidep/agami/ 